\title{
Some Results of Differential Subordination AND DifFERENTIAL Superordination TheOREMS FOR UNIVALENT FunCTIONS DEFINED BY RuscheWEYH DERIVATIVE OPERATOR
}

\author{
Aqeel Ketab AL-KHAFAJI ${ }^{1,2, *}$ \\ ${ }^{1}$ Department of Mathematics, College of Education for Pure Sciences, The University of Babylon, \\ 51002 Babylon, Iraq \\ ${ }^{2}$ Department of Mathematics, College of Education for Pure Sciences-Ibn Al-Haitham, The \\ University of Baghdad, 10071 Baghdad, Iraq \\ *Corresponding Author: Aqeel Ketab AL-KHAFAJI (email: aqeelketab@gmail.com) \\ (Received: 8-April-2019; accepted: 27-June-2019; published: 30-June-2019) \\ DOI: http://dx.doi.org/10.25073/jaec.201932.237
}

\begin{abstract}
The purpose of the present paper is to derive several subordination, superordination results, and sandwich results for the function of the form $f(z)=z+\sum_{n=2}^{\infty} a_{n} z^{n}$ which is univalent in the open unit disc $U=\{z \in \mathbb{C}:|z|<1\}$ by using the Ruscheweyh derivative operator $\mathfrak{R}^{\lambda} f(z)=z+\sum_{n=2}^{\infty} B_{n}(\lambda) a_{n} z^{n}$. Further some of which improve on the previously best-known results achieved for special cases of our work.
\end{abstract}

\section{Keywords}

Univalent Function, Differential Subordination, Differential Superordination, Sandwich Theorem.

\section{INTRODUCTION}

Let $\mathcal{M}=\mathcal{M}(U)$ denote the class of analytic functions in the open unit $\operatorname{disc} U=$ $\{z \in \mathbb{C}:|z|<1\}$. For $\mathrm{n}$ a positive integer and $a \in \mathbb{C}$, let $\mathcal{M}[a, n]$ be the subclass of $\mathcal{M}$ consist- ing of functions of the form:

$$
\begin{array}{r}
f(z)=a+a_{n} z^{n}+a_{n+1} z^{n+1}+\ldots, \\
(a \in \mathbb{C}) .
\end{array}
$$

Also, let $W$ be the subclass of $\mathcal{M}$ consisting of functions of the form:

$$
\begin{array}{r}
f(z)=z+\sum_{n=2}^{\infty} a_{n} z^{n}, \\
\left(a_{n} \geq 0, n \in \mathbb{N}=\{1,2,3, \ldots\}\right)
\end{array}
$$

which are univalent in $U$.

For the function $f \in W$ given by (2) and $g \in W$ defined by:

$$
g(z)=z+\sum_{n=2}^{\infty} b_{n} z^{n} .
$$

The Hadamard product (or convolution) of $f$ and $f$ is defined by:

$$
(f * g)(z)=z+\sum_{n=2}^{\infty} a_{n} b_{n} z^{n}=(g * f)(z) .
$$

For a real number $\lambda>-1$ and $f \in W$. The Ruscheweyh derivative [1] of order $\lambda$ is denoted 
by $\mathfrak{R}^{\lambda} f$ and defined as the following

$$
\begin{aligned}
\mathfrak{R}^{\lambda} f(z) & =f(z) * \frac{1}{(1-z)^{\lambda+1}} \\
& =z+\sum_{n=2}^{\infty} S_{n}(\lambda) a_{n} z^{n},
\end{aligned}
$$

where $S_{n}(\lambda)=\frac{(\lambda+1)(\lambda+2) \ldots(\lambda+n-1)}{(n-1) !}$.

From Eq.(3) we note that:

$$
z\left(\mathfrak{R}^{\lambda} f(z)\right)^{\prime}=(\lambda+1) \mathfrak{R}^{\lambda+1} f(z)-\lambda \mathfrak{R}^{\lambda} f(z) .
$$

In 2005 Bulboacã [2], used the results of Miller and Mocanu [3], they considered certain classes of first order differential superordinatias, as well as superordination-preserving integral operators [2]. In $2004 \mathrm{Ali}$ and others [4] have used the results of Bulboacã [2] to obtain sufficient conditions for certain normalized analytic functions to satisfy

$$
q_{1}(z) \prec \frac{z f^{\prime}(z)}{f(z)} \prec q_{2}(z),
$$

where $q_{1}$ and $q_{2}$ are univalent functions in $U$ with $q_{1}(0)=q_{1}(0)=1$. Tuneski [5] obtained sufficient conditions for starlikeness of $f$ in the terms of the quantity $\frac{z f^{\prime \prime}(z) f(z)}{(f(z))^{2}}$. Recently, Shanmugam and others [6,7] and Goyal and others [8] are obtained some results using sandwich theorem on certain classes of analytic functions. Also see the References [9-11].

The main object of this work is to find sufficient conditions for a certain normalized analytic function $f$ to obtaining and proving several subordination, superordination results and some results depending on sandwich theorem. The analytic function $f$ has the form $f(z)=$ $z+\sum_{n=2}^{\infty} a_{n} z^{n}$ which is univalent in the open unit disc $U=\{z \in \mathbb{C}:|z|<1\}$

$$
l_{1}(z) \prec\left(\frac{\mathfrak{R}^{\lambda} f(z)}{z}\right)^{\tau} \prec l_{2}(z),
$$

and

$$
l_{1}(z) \prec\left(\frac{\beta\left(\mathfrak{R}^{\lambda+1} f(z)\right)+(1-\beta) \mathfrak{R}^{\lambda} f(z)}{z}\right)^{\tau} \prec l_{2}(z),
$$

where $l_{1}$ and $l_{2}$ are given univalent functions in $U$ with $l_{1}(0)=l_{1}(0)=1$.
In order to prove our subordination and superordination we need the following definition and lemmas.

Definition 1.1: [3] If $f, g \in \mathcal{M}(U)$, we say that $f$ is subordinate to $g$ or $g$ is said to be superordinate to $f$, written symbolically $f(z) \prec$ $g(z)$ if there exists a Schwarz function $w$, which is analytic in $U$ with $w(z)=0$ and $|w(z)|<1$ for all $z \in U$, such that $f(z)=g(w(z)), z \in U$. Furthermore, if the function $g$ is univalent in $U$, then we have the following equivalence

$f(z) \prec g(z) \Longleftrightarrow f(0)=g(0)$ and $f(U) \subset g(U)$.

Definition 1.2: [3] Let $\psi: \mathbb{C}^{3} \times U \rightarrow \mathbb{C}$, and $h(z)$ be univalent in $U$. If $k(z)$ is analytic in $U$ and satisfying the second order differential subordination:

$$
\psi\left(k(z), z k^{\prime}(z), z^{2} k^{\prime \prime}(z) ; z\right) \prec h(z),
$$

then $k(z)$ is a solution of the differential subordination (5). The univalent function $q(z)$ is called a dominant of the solution of the differential subordination (5) if $k(z) \prec q(z)$ for all $k(z)$ satisfying (5). A univalent dominant $\tilde{q}$ that satisfying $\tilde{q} \prec q$ for all dominants of $(5)$ is called the beast dominant.

Definition 1.3: [3] Let $\psi: \mathbb{C}^{3} \times U \rightarrow$ $\mathbb{C}$, and $h(z)$ be univalent in $U$. If $k(z)$ and $\psi\left(k(z), z k^{\prime}(z), z^{2} k^{\prime \prime}(z) ; z\right)$ are univalent in $U$ and if $k(z)$ satisfies the second order differential superordination:

$$
h(z) \prec \varphi\left(k(z), z k^{\prime}(z), z^{2} k^{\prime \prime}(z) ; z\right)
$$

then $k(z)$ is a solution of the differential superordination (6). An analytic function $q(z)$ is called a subordinant of the solutions of the differential superordination (6) if $q(z) \prec k(z)$ for all $k(z)$ satisfying (6). A univalent subordinant $\tilde{q}$ that satisfy $q \prec \tilde{q}$ for all subordinants of (6) is called the beast subordinant.

Definition 1.4 [3] Let $Q$ be the set of all functions $f$ that are analytic and injective on $\bar{U} \backslash E(f)$, where

$$
E(f)=\left\{\xi \in \partial U: \lim _{Z \rightarrow \xi} f(z)=\infty\right\},
$$

and are such that $f^{\prime}(\xi) \neq 0$ for $\xi \in \partial U \backslash E(f)$. 
Lemma 1.1 [3] Let $q(z)$ be convex univalent function in the open unit disk $U$ and $\psi, t \in$ $\mathbb{C} \backslash\{0\}$ with

$$
\operatorname{Re}\left(1+\frac{z q^{\prime \prime}(z)}{q^{\prime}(z)}+\frac{\psi}{t}\right)>0 .
$$

If $p(z)$ is analytic in $U$ and

$$
\psi p(z)+t z p^{\prime}(z) \prec \psi q(z)+t z q^{\prime}(z),
$$

then $p(z) \prec q(z)$, and $q(z)$ is the best dominant for $(7)$.

Lemma 1.2 [3] Let $q(z)$ be univalent function in the open unit disk $U$ and let $\theta$ and $\varphi$ be analytic in a domain $D$ containing $q(U)$ with $\varphi(w) \neq 0$ when $w \in q(U)$. Set

$$
Q(z)=z q^{\prime}(z) \varphi(q(z))
$$

and

$$
h(z)=\theta(q(z))+Q(z)
$$

Suppose that

(i) $Q$ is starlike univalent in $U$.

(ii) $\operatorname{Re}\left(\frac{z h^{\prime}(z)}{Q(z)}\right)>0$ for $z \in U$.

If $p(z)$ is analytic with $p(0)=q(0), p(U) \subseteq D$ and

$\theta(p(z))+z p^{\prime}(z) \varphi(p(z)) \prec \theta(q(z))+z q^{\prime}(z) \varphi(q(z))$

then $p(z) \prec q(z)$, and $q(z)$ is the best dominant for $(8)$.

Lemma 1.3 [3] Let $q(z)$ be convex univalent function in the open unit disk $U$ and $\alpha \in \mathbb{C}, \beta \in$ $\mathbb{C} \backslash\{0\}$ with

$$
\operatorname{Re}\left(1+\frac{z q^{\prime \prime}(z)}{q^{\prime}(z)}\right)>\max \left\{0,-\operatorname{Re}\left(\frac{\alpha}{\beta}\right)\right\} .
$$

If $p(z)$ is analytic in $U$ and

$$
\alpha p(z)+\beta z p^{\prime}(z) \prec \alpha q(z)+\beta z q^{\prime}(z),
$$

then $p(z) \prec q(z)$, and $q(z)$ is the best dominant for (9).

Lemma 1.4 [3] Let $q(z)$ be convex function in the open unit disk $U$ and $\beta \in \mathbb{C}$. Further assume that $\operatorname{Re}(\beta)>0$. If $p(z) \in H[q(z), 1]$ and $p(z)+$ $\beta z q^{\prime}(z)$ is univalent in $U$, then

$$
q(z)+\beta z q^{\prime}(z) \prec p(z)+\beta z p^{\prime}(z)
$$

then $q(z) \prec p(z)$, and $q(z)$ is the best subordinant for (10).

Lemma 1.5 [3] Let $q(z)$ be convex univalent function in the open unit disk $U$ and let $\theta$ and $\varphi$ be analytic in a domain $D$ containing $q(U)$. Suppose that

(i) $\operatorname{Re}\left(\frac{\theta^{\prime}(q(z))}{\varphi(q(z))}\right)>0$, for $z \in U$.

(ii) $z q^{\prime}(z) \varphi(q(z))$ is starlike univalent in $U$.

If $p(z) \in H[q(0), 1] \cap Q$, with $p(U) \subseteq D$, and $\theta(p(z))+z p^{\prime}(z) \varphi(p(z))$ is univalent in $U$, and $\theta(q(z))+z q^{\prime}(z) \varphi(q(z)) \prec \theta(p(z))+z p^{\prime}(z) \varphi(p(z))$ then $q(z) \prec p(z)$, and $q(z)$ is the best subordinant for (11).

\section{Subordination Results for $\mathfrak{R}^{\lambda} \boldsymbol{f}(\boldsymbol{z})$}

Theorem 2.1: Let $l$ be a convex univalent in $U$ with $l(0)=1, \tau>0,0 \neq \vartheta \in \mathbb{C}$ and suppose that $l$ satisfies

$$
\operatorname{Re}\left\{1+\frac{z l^{\prime \prime}(z)}{l^{\prime}(z)}\right\}>\max \left\{0,-\operatorname{Re}\left(\frac{\tau}{\vartheta}\right)\right\} .
$$

If $f(z) \in W$, satisfies the subordination:

$$
\begin{array}{r}
{\left[1+\vartheta(\lambda+1)\left(\frac{\mathfrak{R}^{\lambda+1} f(z)}{\mathfrak{R}^{\lambda} f(z)}-1\right)\right]\left(\frac{\mathfrak{R}^{\lambda} f(z)}{z}\right)^{\tau}} \\
\prec l(z)+\frac{\vartheta}{\tau} z l^{\prime}(z),(13)
\end{array}
$$

then

$$
\left(\frac{\mathfrak{R}^{\lambda} f(z)}{z}\right)^{\tau} \prec l(z),
$$

and $l(z)$ is the best dominant for (13).

Proof: define the function $m$ by:

$$
m(z)=\left(\frac{\mathfrak{R}^{\lambda} f(z)}{z}\right)^{\tau} .
$$


Differentiating Eq.(15) logarithmically with re- if $f(z) \in W$, satisfies the subordination: spect to $z$, we obtain:

$$
\frac{z m^{\prime}(z)}{m(z)}=\tau\left(\frac{z\left(\mathfrak{R}^{\lambda} f(z)\right)^{\prime}}{\mathfrak{R}^{\lambda} f(z)}-1\right)
$$

$$
\begin{array}{r}
\left\{1+\vartheta(\lambda+1)\left(\frac{\mathfrak{R}^{\lambda+1} f(z)}{\mathfrak{R}^{\lambda} f(z)}-1\right)\right\}\left(\frac{\mathfrak{R}^{\lambda} f(z)}{z}\right)^{\tau} \\
\prec \frac{1+z}{1-z}+\frac{2 \vartheta z}{\tau(1-z)^{2}}, \quad(17)
\end{array}
$$

From Eq.(4), we obtain:

$$
\frac{z m^{\prime}(z)}{m(z)}=\tau(\lambda+1)\left(\frac{z \mathfrak{R}^{\lambda+1} f(z)}{\mathfrak{R}^{\lambda} f(z)}-1\right) .
$$

then

$$
\left(\frac{\mathfrak{R}^{\lambda} f(z)}{z}\right)^{\tau} \prec \frac{1+z}{1-z},
$$

Therefore,

$\frac{z m^{\prime}(z)}{\tau}=(\lambda+1)\left(\frac{\mathfrak{R}^{\lambda} f(z)}{z}\right)^{\tau}\left(\frac{z \mathfrak{R}^{\lambda+1} f(z)}{\mathfrak{R}^{\lambda} f(z)}-1\right)$. and $l(z)=\frac{1+z}{1-z}$ is the best dominant for (17).

Theorem 2.2: Let $l$ be a convex univalent in $U$ with $l(0)=1$ and $l(z) \neq 0$ for all $z \in U$, and suppose that $l$ satisfies:

The subordination (13) from the hypothesis becomes:

$$
l(z)+\frac{\vartheta}{\tau} z l^{\prime}(z) \prec m(z)+\frac{\vartheta}{\tau} z m^{\prime}(z) .
$$

An application of Lemma 1.3, with $\beta=\frac{\vartheta}{\tau}$ and $\alpha=1$, the proof of Theorem 2.1, is completed.

Putting $m(z)=\frac{1+A z}{1+B z}$ where $-1 \leq B<A \leq 1$, in Theorem 2.1, we obtain on the next result.

Corollary 2.1: Let $-1 \leq B<A \leq 1, \tau>$ $0,0 \neq \vartheta \in \mathbb{C}$ and

$$
\operatorname{Re}\left\{\frac{1-B z}{1+B z}\right\}>\max \left\{0,-\operatorname{Re}\left(\frac{\tau}{\vartheta}\right)\right\},
$$

$$
\begin{aligned}
\operatorname{Re}\{1 & +\frac{\nu \xi}{\vartheta}+\frac{\mu(\xi+1)}{\vartheta} l(z) \\
& \left.+(\xi-1) \frac{z l^{\prime}(z)}{l(z)}+\frac{z l^{\prime \prime}(z)}{l^{\prime}(z)}\right\}>0
\end{aligned}
$$

where $\xi, \mu, \nu \in \mathbb{C}, 0 \neq \vartheta \in \mathbb{C}$ and $z \in U$.

Suppose that $z(l(z))^{\xi-1} l^{\prime}(z)$ is starlike univalent in $\mathrm{U}$.

If $f(z) \in W$, satisfies the subordination:

$$
\begin{aligned}
\mathcal{G}(\xi, \nu, \mu, \beta, \lambda, \vartheta ; z) \prec & (\nu+\mu l(z))(l(z))^{\xi} \\
& +\vartheta z(l(z))^{\xi-1} l^{\prime}(z),
\end{aligned}
$$

where

if $f(z) \in W$, satisfies the subordination:

$$
\mathcal{G}(\xi, \nu, \mu, \beta, \lambda, \vartheta ; z)
$$$$
\left\{1+\vartheta(\lambda+1)\left(\frac{\mathfrak{R}^{\lambda+1} f(z)}{\mathfrak{R}^{\lambda} f(z)}-1\right)\right\}\left(\frac{\mathfrak{R}^{\lambda} f(z)}{z}\right)^{\tau}
$$$$
\prec \frac{1+A z}{1+B z}+\frac{\vartheta}{\tau} \frac{(A-B) z}{(1+B z)^{2}},
$$

then

$$
\left(\frac{\mathfrak{R}^{\lambda} f(z)}{z}\right)^{\tau} \prec \frac{1+A z}{1+B z},
$$

and $l(z)=\frac{1+A z}{1+B z}$ is the best dominant for (16). In Corollary 2.1, if the values of A and B are $1,-1$; respectively, we obtain the following result:

Corollary 2.2: Let $A=1, B=-1, \tau>$ $0,0 \neq \vartheta \in \mathbb{C}$ and

$$
\max \left\{0,-\operatorname{Re}\left(\frac{\tau}{\vartheta}\right)\right\}<1,
$$

then

$$
\left(\frac{\beta \mathfrak{R}^{\lambda} f(z)+(1-\beta) \mathfrak{R}^{\lambda+1} f(z)}{z}\right)^{\tau} \prec l(z),
$$

and $l(z)$ is the best dominant for (19). 
Proof: Define the function $m$ by:

$$
m(z)=\left(\frac{\beta \mathfrak{R}^{\lambda} f(z)+(1-\beta) \mathfrak{R}^{\lambda+1} f(z)}{z}\right)^{\tau}
$$

By setting

$$
\begin{aligned}
\psi(\mathcal{B})=(\nu+\mu \mathcal{B}) \mathcal{B}^{\xi} \text { and } \phi(\mathcal{B})=\vartheta(\mathcal{B})^{\xi-1} & \\
0 & \neq \mathcal{B} \in \mathbb{C}
\end{aligned}
$$

we see also that $\psi(\mathcal{B})$ is analytic in $\mathbb{C}, \phi(\mathcal{B})$ is analytic in $\mathbb{C}-\{0\}$ and that $\phi(\mathcal{B}) \neq 0$. Also we obtain

$$
\wp(z)=z l^{\prime}(z) \phi(l(z))=\vartheta z(l(z))^{\xi-1} l^{\prime}(z),
$$

and

$$
\begin{aligned}
g(z) & =\psi(l(z))+\wp(z) \\
& =(\nu+\mu l(z))(l(z))^{\xi}+\vartheta z(l(z))^{\xi-1} l^{\prime}(z) .
\end{aligned}
$$

Since $\left[z(l(z))^{\xi-1} l^{\prime}(z)\right]$ starlike univalent, then $\wp(z)$ is starlike univalent in $U$,

$$
\begin{aligned}
\operatorname{Re}\left\{\frac{z g^{\prime}(z)}{\wp(z)}\right\} & =\operatorname{Re}\left\{1+\frac{\nu \xi}{\vartheta}+\frac{\mu(\xi+1)}{\vartheta} l(z)\right. \\
& \left.+(\xi-1) \frac{z l^{\prime}(z)}{l(z)}+\frac{z l^{\prime \prime}(z)}{l^{\prime}(z)}\right\}>0 .
\end{aligned}
$$

The following equation can be obtained by a straight word computation:

$$
\begin{aligned}
& (\nu+\mu m(z))(m(z))^{\xi}+\vartheta z(m(z))^{\xi-1} m^{\prime}(z) \\
= & \mathcal{G}(\xi, \nu, \mu, \beta, \lambda, \vartheta ; z),
\end{aligned}
$$

where $\mathcal{G}(\xi, \nu, \mu, \beta, \lambda, \vartheta ; z)$ is given by $(20)$.

From (19) and Eq. (23), we have the following subordination:

$$
\begin{aligned}
& (\nu+\mu p(z))(m(z))^{\xi}+\vartheta z(m(z))^{\xi-1} m^{\prime}(z) \\
\prec & (\nu+\mu l(z))(l(z))^{\xi}+\vartheta z(l(z))^{\xi-1} l^{\prime}(z), \quad(24)
\end{aligned}
$$

therefore, by using Lemma 1.2, we get on:

$m(z) \prec l(z)$ and $l(z)$ the best dominant of (19)

Putting $l(z)=e^{\delta z},|\delta| \leq 1$ in Theorem 2.2, we obtain the following result:

Corollary 2.3: Let $|\delta| \leq 1$ and

$$
\operatorname{Re}\left\{1+\frac{\nu \xi}{\vartheta}+\frac{\mu(\xi+1)}{\vartheta} e^{\delta z}+z \delta \xi\right\}>0,
$$

where $\xi, \mu, \nu \in \mathbb{C}, 0 \neq \vartheta \in \mathbb{C}$ and $z \in U$.

If $f(z) \in W$, satisfy the subordination:

$$
\begin{aligned}
\mathcal{G}(\xi, \nu, \mu, \beta, \lambda, \vartheta ; z) \prec & \left(\nu+\mu e^{\delta z}\right) e^{\xi \delta z} \\
& +\vartheta \delta z e^{(\xi-1) \delta z} e^{\delta z},
\end{aligned}
$$

where $\mathcal{G}(\xi, \nu, \mu, \beta, \lambda, \vartheta ; z)$ is given by $(20)$, then

$$
\left(\frac{\beta \Re^{\lambda} f(z)+(1-\beta) \mathfrak{R}^{\lambda+1} f(z)}{z}\right)^{\tau} \prec e^{\delta z},
$$

and $e^{\delta z}$ is the best dominant for (25).

Hence, for the particular case $\delta=\beta=1$, we have the following result:

Corollary 2.4: Let $\delta=\beta=1$ and

$$
\operatorname{Re}\left\{1+\frac{\nu \xi}{\vartheta}+\frac{\mu(\xi+1)}{\vartheta} e^{z}+z \xi\right\}>0,
$$

where $\xi, \mu, \nu \in \mathbb{C}, 0 \neq \vartheta \in \mathbb{C}$ and $z \in U$.

If $f(z) \in W$, satisfies the subordination:

$$
\mathcal{G}(\xi, \nu, \mu, 1, \lambda, \vartheta ; z) \prec\left(\nu+\mu e^{z}+\vartheta z\right) e^{\xi z},
$$

Where $\mathcal{G}(\xi, \nu, \mu, 1, \lambda, \vartheta ; z)$ is given by $(20)$, then

$$
\left(\frac{\mathfrak{R}^{\lambda} f(z)}{z}\right)^{\tau} \prec e^{z},
$$

and $e^{z}$ is the best dominant for (26) .

\section{Superordinations results for $\mathfrak{R}^{\lambda} \boldsymbol{f}(\boldsymbol{z})$}

Theorem 3.1: Let $l$ be a convex univalent in $U$ with $l(0)=1, \tau>0, \operatorname{Re}(\vartheta)>0$. Let $f(z) \in$ $W$, satisfies $\left(\frac{\mathfrak{R}^{\lambda} f(z)}{z}\right)^{\tau} \in \mathcal{M}[l(0), 1] \cap Q$, and $\left[1+\vartheta(\lambda+1)\left(\frac{\mathfrak{R}^{\lambda+1} f(z)}{\mathfrak{R}^{\lambda} f(z)}-1\right)\right]\left(\frac{\mathfrak{R}^{\lambda} f(z)}{z}\right)^{\tau}$

be univalent in $U$. If

$$
\begin{aligned}
l(z)+\frac{\vartheta}{\tau} z l^{\prime}(z) \prec & {\left[1+\vartheta(\lambda+1)\left(\frac{\mathfrak{R}^{\lambda+1} f(z)}{\mathfrak{R}^{\lambda} f(z)}-1\right)\right] } \\
& \times\left(\frac{\mathfrak{R}^{\lambda} f(z)}{z}\right)^{\tau},
\end{aligned}
$$


then

$$
l(z) \prec\left(\frac{\mathfrak{R}^{\lambda} f(z)}{z}\right)^{\tau},
$$

and $l(z)$ is the best subordinant for $(27)$.

Proof: Define the function $m$ by:

$$
m(z)=\left(\frac{\mathfrak{R}^{\lambda} f(z)}{z}\right)^{\tau} .
$$

Differentiating (29) logarithmically with respect to $z$, we obtain:

$$
\frac{z m^{\prime}(z)}{m(z)}=\tau\left(\frac{z\left(\mathfrak{R}^{\lambda} f(z)\right)^{\prime}}{\mathfrak{R}^{\lambda} f(z)}-1\right) .
$$

So by using Eq.(4), from Eq.(29), we obtain:
In Corollary 3.1, if the values of $\mathrm{A}$ and $\mathrm{B}$ are $1,-1$; respectively, we obtain the following result:

Corollary 3.2: Let $A=1, B=-1, \tau>$ $0,0 \neq \vartheta \in \mathbb{C}$ and $\operatorname{Re}\{\vartheta\}>0$, let $f(z) \in W$, satisfies $\left(\frac{D^{\lambda} f(z)}{z}\right)^{\tau} \in \mathcal{M}[l(0), 1] \cap Q$, and let

$$
\left[1+\vartheta(\lambda+1)\left(\frac{\mathfrak{R}^{\lambda+1} f(z)}{\mathfrak{R}^{\lambda} f(z)}-1\right)\right]\left(\frac{\mathfrak{R}^{\lambda} f(z)}{z}\right)^{\tau},
$$

be univalent in $U$. If

$$
\begin{aligned}
& \frac{1+z}{1-z}+\frac{2 \vartheta z}{\tau(1-z)^{2}} \\
\prec & {\left[1+\vartheta(\lambda+1)\left(\frac{\mathfrak{R}^{\lambda+1} f(z)}{\mathfrak{R}^{\lambda} f(z)}-1\right)\right]\left(\frac{\mathfrak{R}^{\lambda} f(z)}{z}\right)^{\tau}, }
\end{aligned}
$$

$$
\left[1+\vartheta(\lambda+1)\left(\frac{\mathfrak{R}^{\lambda+1} f(z)}{\mathfrak{R}^{\lambda} f(z)}-1\right)\right]\left(\frac{\mathfrak{R}^{\lambda} f(z)}{z}\right)^{\tau} \text { then } \quad \frac{1+z}{1-z} \prec\left(\frac{\mathfrak{R}^{\lambda} f(z)}{z}\right)^{\tau}
$$$$
=m(z)+\frac{\vartheta}{\tau} z m^{\prime}(z) \text {. }
$$

From subordination (27), we have:

$$
l(z)+\frac{\vartheta}{\tau} z l^{\prime}(z) \prec m(z)+\frac{\vartheta}{\tau} z m^{\prime}(z)
$$

An application of Lemma 1.4, with $\beta=\frac{\vartheta}{\tau}$, we get the desired result.

Putting $l(z)=\frac{1+A z}{1+B z}$ where $-1 \leq B<A \leq 1$, in Theorem 3.1, we obtain on the next result.

Corollary 3.1: Let $-1 \leq B<A \leq 1, \tau>$ $0,0 \neq \vartheta \in \mathbb{C}$ and $\operatorname{Re}\{\vartheta\}>0$, let $f(z) \in W$, satisfies $\left(\frac{\mathfrak{R}^{\lambda} f(z)}{z}\right)^{\tau} \in \mathcal{M}[l(0), 1] \cap Q$, and let

$$
\left[1+\vartheta(\lambda+1)\left(\frac{\mathfrak{R}^{\lambda+1} f(z)}{\mathfrak{R}^{\lambda} f(z)}-1\right)\right]\left(\frac{\mathfrak{R}^{\lambda} f(z)}{z}\right)^{\tau}
$$

be univalent in $U$. If

$$
\begin{aligned}
& \frac{1+A z}{1+B z}+\frac{\vartheta}{\tau} \frac{(A-B) z}{(1+B z)^{2}} \\
\prec & {\left[1+\vartheta(\lambda+1)\left(\frac{\mathfrak{R}^{\lambda+1} f(z)}{\mathfrak{R}^{\lambda} f(z)}-1\right)\right]\left(\frac{\mathfrak{R}^{\lambda} f(z)}{z}\right)^{\tau}, }
\end{aligned}
$$

then

$$
\frac{1+A z}{1+B z} \prec\left(\frac{\mathfrak{R}^{\lambda} f(z)}{z}\right)^{\tau},
$$

and $l(z)=\frac{1+A z}{1+B z}$ is the best subordinant for (30) . and $l(z)=\frac{1+z}{1-z} \quad$ is the best subordinant for (31).

Next, we prove the following theorem by using Lemma 1.5.

Theorem 3.2: Let $l$ be a convex univalent in $U$ with $l(0)=1$, assume that $l$ satisfies

$$
\operatorname{Re}\left\{\frac{\nu \xi}{\vartheta} l^{\prime}(z)+\frac{\mu(\xi+1)}{\vartheta} l(z) l^{\prime}(z)\right\}>0,
$$

where $\nu, \mu, \xi \in \mathbb{C}, \vartheta \in \mathbb{C}-\{0\}$ and $z \in U$.

and that $z(l(z))^{\xi-1} l^{\prime}(z)$ is starlike univalent in U. Let $f(z) \in W$, satisfies the condition:

$\left(\frac{\beta \mathfrak{R}^{\lambda} f(z)+(1-\beta) \mathfrak{R}^{\lambda+1} f(z)}{z}\right)^{\tau} \in \mathcal{M}[l(0), 1] \cap Q$,

where $(0 \leq \beta \leq 1, \tau>0$ and $z \in U)$,

and $\mathcal{G}(\xi, \nu, \mu, \beta, \lambda, \vartheta ; z)$ is univalent in $U$, where $\mathcal{G}(\xi, \nu, \mu, \beta, \lambda, \vartheta ; z)$ is given by $(20)$. If

$$
\begin{aligned}
& (\nu+\mu q(z))(l(z))^{\xi}+\vartheta z(l(z))^{\xi-1} l^{\prime}(z) \\
\prec & \mathcal{G}(\xi, \nu, \mu, \beta, \lambda, \vartheta ; z)
\end{aligned}
$$

then

$$
l(z) \prec\left(\frac{\beta \Re^{\lambda} f(z)+(1-\beta) \mathfrak{R}^{\lambda+1} f(z)}{z}\right)^{\tau},
$$


and $l(z)$ is the best subordinant for (33).

Proof: Define the function $m$ by:

$$
m(z)=\left(\frac{\beta \Re^{\lambda} f(z)+(1-\beta) \mathfrak{R}^{\lambda+1} f(z)}{z}\right)^{\tau}
$$

By setting

$$
\begin{aligned}
\psi(\mathcal{B})=(\nu+\mu \mathcal{B}) \mathcal{B}^{\xi} \text { and } \phi(\mathcal{B}) & =\vartheta(\mathcal{B})^{\xi-1} \\
0 & \neq \mathcal{B} \in \mathbb{C}
\end{aligned}
$$

we see also that $\psi(\mathcal{B})$ is analytic in $\mathbb{C}, \phi(\mathcal{B})$ is analytic in $\mathbb{C}-\{0\}$ and that $\phi(\mathcal{B}) \neq 0$. Also we get

$$
\wp(z)=z l^{\prime}(z) \phi(l(z))=\vartheta z(l(z))^{\xi-1} l^{\prime}(z) .
$$

And hence, $\wp(z)$ is starlike univalent in $U$ (by assumption),

$$
\begin{array}{r}
\operatorname{Re}\left\{\frac{\psi^{\prime}(z)}{\phi(z)}\right\}=\operatorname{Re}\left\{\frac{\nu \xi}{\vartheta} l^{\prime}(z)\right. \\
\left.+\frac{\mu(\xi+1)}{\vartheta} l(z) l^{\prime}(z)\right\}>0 .
\end{array}
$$

We get on the following Equation, if make a straight word computation:

$$
\begin{aligned}
\mathcal{G}(\xi, \nu, \mu, \beta, \lambda, \vartheta ; z)= & (\nu+\mu p(z))(m(z))^{\xi} \\
& +\vartheta z(m(z))^{\xi-1} m^{\prime}(z)
\end{aligned}
$$

where $\mathcal{G}(\xi, \nu, \mu, \beta, \lambda, \vartheta ; z)$ is given by $(20)$.

From (33) and (36), we have the following relation:

$$
\begin{aligned}
& (\nu+\mu q(z))(l(z))^{\xi}+\vartheta z(l(z))^{\xi-1} l^{\prime}(z) \\
\prec & (\nu+\mu m(z))(m(z))^{\xi}+\vartheta z(m(z))^{\xi-1} m^{\prime}(z),
\end{aligned}
$$

therefore, by using Lemma 1.5, we get on: $m(z) \prec l(z)$ and $l(z)$ the best subordinant (33).

Putting $l(z)=e^{\delta z},|\delta| \leq 1$ in Theorem 3.2, we get the following result:

Corollary 3.3: Let $|\delta| \leq 1$ and

$$
\operatorname{Re}\left\{\frac{\nu \xi \delta}{\vartheta} e^{\delta z}+\frac{\mu(\xi+1) \delta}{\vartheta} e^{2 \delta z}\right\}>0,
$$

where $\xi, \mu, \nu \in \mathbb{C}, 0 \neq \vartheta \in \mathbb{C}$ and $z \in U$.

If $f(z) \in W$, satisfies the superordination:

$\left(\nu+\mu e^{\delta z}\right) e^{\delta \xi z}+\vartheta z \delta e^{\delta(\xi-1) z} \prec \mathcal{G}(\xi, \nu, \mu, \beta, \lambda, \vartheta ; z)$

Where $\mathcal{G}(\xi, \nu, \mu, \beta, \lambda, \vartheta ; z)$ is given by $(20)$,

then

$$
\left(\frac{\beta \mathfrak{R}^{\lambda} f(z)+(1-\beta) \mathfrak{R}^{\lambda+1} f(z)}{z}\right)^{\tau} \prec e^{\delta z},
$$

and $e^{\delta z}$ is the best subordinat for (37).

Hence, in the particular case $\delta=\beta=1$, we have the following result:

Corollary 3.4: Let $\delta=\beta=1$ and

$$
\operatorname{Re}\left\{\frac{\nu \xi}{\vartheta} e^{z}+\frac{\mu(\xi+1)}{\vartheta} e^{2 z}\right\}>0,
$$

where $\xi, \mu, \nu \in \mathbb{C}, 0 \neq \vartheta \in \mathbb{C}$ and $z \in U$.

If $f(z) \in W$, satisfies the superordination:

$$
\left(\nu+\mu e^{z}\right) e^{\xi z}+\vartheta z e^{(\xi-1) z} \prec \mathcal{G}(\xi, \nu, \mu, \beta, \lambda, \vartheta ; z)
$$

where $\mathcal{G}(\xi, \nu, \mu, 1, \lambda, \vartheta ; z)$ is given by $(20)$,

then

$$
\left(\frac{\mathfrak{R}^{\lambda} f(z)}{z}\right)^{\tau} \prec e^{z},
$$

and $e^{z}$ is the best subordinat for (38).

\section{Sandwich results}

Combining Theorem 2.1 with Theorem 3.1 and Theorem 2.2 with Theorem 3.2, we arrive at the following sandwich result.

Theorem 4.1: Let $l_{1}(z)$ and $l_{2}(z)$ be convex univalent functions in $U$ with $l_{1}(0)=l_{2}(0)=$ 1. Let $l_{1}$ and $l_{2}$ satisfies $\operatorname{Re}(\vartheta)>0$ and $R e\left\{1+\frac{z l^{\prime \prime}(z)}{l^{\prime}(z)}\right\}>\max \left\{0,-\operatorname{Re}\left(\frac{\tau}{\vartheta}\right)\right\}$ respectively, where $\tau>0,0 \neq \vartheta \in \mathbb{C}$. Let $f(z) \in W$, satisfies

$$
\left(\frac{\mathfrak{R}^{\lambda} f(z)}{z}\right)^{\tau} \in \mathcal{M}[l(0), 1] \cap Q,
$$

and

$$
\left[1+\vartheta(\lambda+1)\left(\frac{\mathfrak{R}^{\lambda+1} f(z)}{\mathfrak{R}^{\lambda} f(z)}-1\right)\right]\left(\frac{\mathfrak{R}^{\lambda} f(z)}{z}\right)^{\tau}
$$


be univalent in $U$. If

$$
\begin{aligned}
& l_{1}(z)+\frac{\vartheta}{\tau} z l_{1}^{\prime}(z) \\
\prec & {\left[1+\vartheta(\lambda+1)\left(\frac{\mathfrak{R}^{\lambda+1} f(z)}{\mathfrak{R}^{\lambda} f(z)}-1\right)\right]\left(\frac{\mathfrak{R}^{\lambda} f(z)}{z}\right)^{\tau} } \\
\prec & l_{2}(z)+\frac{\vartheta}{\tau} z l_{2}^{\prime}(z),
\end{aligned}
$$

then

$$
l_{1}(z) \prec\left(\frac{\mathfrak{R}^{\lambda} f(z)}{z}\right)^{\tau} \prec l_{2}(z)
$$

and $l_{1}(z), l_{2}(z)$ are respectively, the best subordinant and the best dominant.

Theorem 4.2: Let $l_{1}(z)$ and be $l_{2}(z)$ a convex univalent functions in $U$ with $l_{1}(0)=l_{2}(0)=1$. Let $l_{1}$ and $l_{2}$ satisfies the Inequality (15) and the Inequality (29) respectively, and let $f(z) \in W$, satisfies the condition:

$$
\left(\frac{\beta \Re^{\lambda} f(z)+(1-\beta) \mathfrak{R}^{\lambda+1} f(z)}{z}\right)^{\tau} \in \mathcal{M}[1,1] \cap Q,
$$

where $(0 \leq \beta \leq 1, \tau>0$ and $z \in U)$,

and $\mathcal{G}(\xi, \nu, \mu, \beta, \lambda, \vartheta ; z)$ is univalent in $U$, where $\mathcal{G}(\xi, \nu, \mu, \beta, \lambda, \vartheta ; z)$ is given by $(20)$. If

$$
\begin{aligned}
& \left(\nu+\mu l_{1}(z)\right)\left(l_{1}(z)\right)^{\xi}+\vartheta z\left(l_{1}(z)\right)^{\xi-1} l_{1}^{\prime}(z) \\
\prec & \mathcal{G}(\xi, \nu, \mu, \beta, \lambda, \vartheta ; z) \\
\prec & \left(\nu+\mu l_{2}(z)\right)\left(l_{2}(z)\right)^{\xi}+\vartheta z\left(l_{2}(z)\right)^{\xi-1} l_{2}^{\prime}(z),
\end{aligned}
$$

then

$l_{1}(z) \prec\left(\frac{\beta \Re^{\lambda} f(z)+(1-\beta) \mathfrak{R}^{\lambda+1} f(z)}{z}\right)^{\tau} \prec l_{2}(z)$

and $l_{1}(z), l_{2}(z)$ are respectively, the best subordinant and the best dominant.

\section{References}

[1] Ruscheweyh, S. (1975). New criteria for univalent functions. Proc. Amer. Math. Soc. 49, 109-115.

[2] Bulboaca, E. (2005). Differential subordinations and superordinations, Recent Results, House of Scientific Book Publ., ClujNapoca.

[3] Miller, S. S., \& MocAnu, P. T. (2003). Subordinates of Differential Superordinations, Complex Var. Theory Appl., 48(10), 815826.
[4] Ali, R. M., Revichandran, V., Khan, M. H., \& Subromanian, K. G. (2004). Differential sandwich theorems for certain analytic function. For East J. Math. 15(1), 87-94.

[5] Tuneski, N. (2000). On certain sufficient conditions for starlikeness. Int. J. Math. Sci., 23(8), 521-527.

[6] Shanmugam, T. N., Ravichandran V., \& Sivasubramanian, S. (2006). Differential sandwich theorems for some subclasses of analytic functions. Aust. J. Math. Anal. Appl., 3(1), 1-11.

[7] Shanmugam, T. N., Sivasubramanian, S., \& Silverman, H. (2006). On sandwich theorems for some classes of analytic functions. Int. J. Math. Sci. Article ID 29684, 1-13.

[8] Goyal, S. P., Goswami, P., \& Silverman, H. (2008). Subordination and superordination result for a class of analytic multivalent functions. Int. J. Math. Sci. Article ID 561638, 1-12.

[9] AL-khafaji, A. K., Atshan, W. G., \& Abed, S. S. (2018). On the Generalization of a Class of Harmonic Univalent Functions Defined by Differential Operator. Mathemat$i c s, 6(12), 312$.

[10] AL-khafaji, A. K., Atshan, W. G., \& Abed, S. S. (2018). On a Differential Subordination of a Certain Subclass of Univalent Functions. Journal of Kufa for Mathematics and Computer. 5(3) 11-16.

[11] Bulboacã, T. (2002). Classes of first order differential superordination. Demonstration Mathematica, 35(2), 287-292.

\section{About Authors}

Aqeel Ketab AL-KHAFAJI received BSc in mathematics from department of mathematics, College of Education for Pure Sciences-Ibn Al-Haitham, The University of Baghdad, 10071 Baghdad, Iraq in 1998, MSc in mathematics from department of mathematics, College of Education for Pure Sciences, The University of Babylon, 51002 Babylon, Iraq, in 2001, 
and $\mathrm{PhD}$ in mathematics (Complex Analysis) from department of mathematics, College of Education for Pure Sciences-Ibn Al-Haitham, The University of Baghdad, 10071 Baghdad, Iraq, in 2019. Dr. Aqeel Ketab AL-khafaji has published 12 papers and 3 contributions in scientific conferences. 